\title{
Synovial Alpha-defensin at Reimplantation in Two-stage Revision Arthroplasty to Rule Out Persistent Infection
}

\author{
CHARLOTTE BIELEFELD ${ }^{1,2}$, HARALD ENGLER ${ }^{3}$, MARCUS JÄGER ${ }^{1,2}$, \\ ALEXANDER WEGNER $^{1,2}$, DENNIS WASSENAAR ${ }^{1,2}$ and ANDRE BUSCH ${ }^{1,2}$ \\ ${ }^{1}$ Department of Orthopaedics, Trauma and Reconstructive Surgery, \\ St. Marien Hospital, Mülheim an der Ruhr, Germany; \\ ${ }^{2}$ Department of Orthopaedics and Trauma Surgery, University of Duisburg-Essen, Mülheim an der Ruhr, Germany; \\ ${ }^{3}$ Institute of Medical Psychology and Behavioral Immunobiology, \\ University Hospital Essen, University of Duisburg-Essen, Essen, Germany
}

\begin{abstract}
Background/Aim: Owing to the lack of a diagnostic gold standard, ruling out persistent periprosthetic joint infection (PJI) before second-stage surgery in the setting of two-stage revision arthroplasty constitutes a major challenge. We evaluated if the alpha-defensin-1 (AD-1) test could predict successful infection eradication before reimplantation of a new prosthesis. Patients and Methods: Our prospective study included 20 patients who underwent two-stage revision arthroplasty for treatment of PJI. A standard quantitative enzyme AD-1 immunoassay of synovial fluid, the synovial leukocyte esterase test and routine laboratory blood testing were performed prior to explantation and reimplantation. Treatment failure was defined according to the Delphi-based consensus criteria after a minimum follow-up of 1 year. Results: A $15 \%$ of our patients met the Delphi Criteria within 1 year. None of the markers investigated were significantly different in patients with and without reinfection. Conclusion: Further research is necessary to identify biomarkers more suitable for indicating persistent infection before reimplantation.
\end{abstract}

Periprosthetic joint infection (PJI) is a serious complication after total joint arthroplasty (TJA), with considerable mortality rates $(1,2)$. Despite intensive research, diagnosis of

This article is freely accessible online.

Correspondence to: Marcus Jäger, Department of Orthopaedics, Trauma and Reconstructive Surgery St. Marienhospital Mülheim an der Ruhr, Department of Orthopaedics and Trauma Surgery University of Duisburg-Essen, Kaiserstraße 50, 45468 Mülheim an der Ruhr, Germany. Tel: +49 2083052202, e-mail: M.Jaeger@contilia.de

Key Words: Alpha-defensin, persistent PJI, reimplantation, spacer, arthroplasty, synovial fluid.
PJI remains challenging. In 2018, Parvizi et al. published a recommendation for reliable diagnosis of PJI (3). There is a trend in diagnosing PJI by testing biomarkers in the synovial fluid $(4,5)$. As it turned out, the synovial alpha-defensin-1 (AD-1) test showed the highest accuracy to diagnose PJI (6).

Two-stage surgery has traditionally been considered the gold standard for PJI treatment (7). After removal of the infectious implant, an aggressive debridement is undertaken, including a complete synovectomy and the application of an antibiotic-loaded cement spacer. Afterwards, patients are treated for several weeks with systemic antibiotics. Secondstage surgery includes removal of the cement spacer, followed by soft and hard tissue debridement and placement of a new TJA (8). Before reimplantation, it is crucial to rule out persistent infection (9). Diaz-Ledezma et al. (2013) published a consensus regarding what constitutes successful treatment of a PJI. Treatment success was described if eradication of infection was reached, no subsequent surgical intervention was performed and no mortality related to PJI occurred (10).

However, there is a lack of reliable laboratory tests to determine whether infection has been successfully eradicated $(11,12)$. Until now, only one study evaluated the benefit of AD-1 testing before reimplantation in a two-stage revision (9). The purpose of the present study was to determine whether persistent infection can be reliably detected by AD- 1 test.

\section{Patients and Methods}

The study was approved by the local institutional review board (188042-BO) and performed in accordance with the declaration of Helsinki. The patients for this prospective study were recruited from the Department of Orthopedics and Trauma Surgery of the University of Duisburg-Essen, Germany.

Patients were included in the study if two-stage revision arthroplasty was performed due to PJI, with intermittent implantation of an antibiotic-loaded cement spacer (3). Testing of the synovial fluid for AD-1 levels was performed prior to explantation and 
in vivo $35: 1073-1081(2021)$

Table I. Changes between the two stages for the overall patient collective.

\begin{tabular}{lccc}
\hline Test result & First stage & Second stage & $p$-Value \\
\hline Mean synovial AD-1 (ng/ml) & $4,890.99(154.43-19,796.20)$ & $3,711.03 \pm 2,174.38$ & 0.35 \\
Mean fibrinogen $(\mathrm{mg} / \mathrm{dl})$ & $481.35 \pm 136.69$ & $373.35 \pm 65.07$ & 0.025 \\
Mean serum WBC/ $\mu \mathrm{l}$ & $7,959 \pm 2,261$ & $6,713 \pm 2,206$ & 0.035 \\
Mean serum CRP $(\mathrm{mg} / \mathrm{dl})$ & $6.15(0-31.3)$ & $2.24(0.5-7.5)$ & 0.01 \\
\hline
\end{tabular}

Table II. Test validity of inflammatory blood parameters for persistent periprosthetic joint infection.

\begin{tabular}{|c|c|c|c|c|c|c|}
\hline Parameter (Mean) & Re-infection & Infection-free & $\begin{array}{c}\text { AUC } \\
(95 \% \mathrm{CI})\end{array}$ & $\begin{array}{l}\text { Cut-off } \\
\text { level }\end{array}$ & $\begin{array}{l}\text { Sensitivity } \\
(95 \% \mathrm{CI})\end{array}$ & $\begin{array}{l}\text { Specificity } \\
(95 \% \mathrm{CI})\end{array}$ \\
\hline Fibrinogen $(\mathrm{mg} / \mathrm{dl})$ & $299.33 \pm 30.07$ & $386.41 \pm 60.88$ & $\begin{array}{c}0.157 \\
(0-0.331)\end{array}$ & 400 & $\begin{array}{c}0 \% \\
(0-56.2 \%)\end{array}$ & $\begin{array}{c}41.2 \% \\
(21.6-64.0 \%)\end{array}$ \\
\hline $\mathrm{WBC} / \mu \mathrm{l}$ & $5,983 \pm 2,439$ & $6,842 \pm 2,218$ & $\begin{array}{c}0.373 \\
(0.012-0.733)\end{array}$ & 8,260 & $\begin{array}{c}33.3 \% \\
(1.7-88.2 \%)\end{array}$ & $\begin{array}{c}70.6 \% \\
(46.9-86.7 \%)\end{array}$ \\
\hline $\mathrm{CRP}(\mathrm{mg} / \mathrm{dl})$ & $<0.5$ & $\begin{array}{c}2.11 \\
(0.5-7.5)\end{array}$ & $\begin{array}{c}0.029 \\
(0-0.102)\end{array}$ & 3.85 & $\begin{array}{c}0 \% \\
(0-56.2 \%)\end{array}$ & $\begin{array}{c}88.2 \% \\
(65.7-97.9 \%)\end{array}$ \\
\hline
\end{tabular}

Table III. Causative organisms of initial periprosthetic joint infection.

\begin{tabular}{lcc}
\hline Organism cultured at first stage & Frequency & Percent \\
\hline Staphylococcus aureus & 4 & 20 \\
Staphylococcus epidermidis & 3 & 15 \\
Staphylococcus haemolyticus & 1 & 5 \\
Propionibacterium spp. & 1 & 5 \\
Pseudomonas aeruginosa & 1 & 5 \\
Klebsiella pneumoniae & 1 & 5 \\
Serratia marcescens & 1 & 5 \\
Enterococcus faecalis & 1 & 5 \\
Escherichia coli & 1 & 5 \\
Enterococcus faecium & 1 & 5 \\
Proteus mirabilis & 1 & 5 \\
Staphylococcus epidermidis & 1 & 5 \\
+Pseudomonas aeruginosa & & \\
Staphylococcus epidermidis & 1 & 5 \\
+Staphylococcus aureus & & 10 \\
Culture-negative PJI & 2 & \\
\hline
\end{tabular}

reimplantation. The minimum follow-up was 12 months. From July 2018 to August 2019, 20 patients (12 hips, 8 knees) met all criteria and were included in the study. The cohort included 13 women and 7 men who had a mean age of 72 years (range $=47-89$ years) and mean BMI of $29.3 \mathrm{~kg} / \mathrm{m}^{2}$ (range $\left.=19.1-42.5 \mathrm{~kg} / \mathrm{m}^{2}\right)$.

Besides AD-1 testing, the synovial leucocyte esterase (LE) reaction was carried out for each patient by applying a drop of centrifuged synovial fluid on a LE test strip (DFI, One Step DUS 10). Joint fluid and tissue samples were collected during reimplantation for further microbiological investigation. Serum Creactive protein (CRP), white blood cell count (WBC) and fibrinogen were determined in the blood drawn preoperatively.
Serum CRP was analyzed by immune turbidimetry (Centaur, Siemens, Germany) (normal value $<0.5 \mathrm{mg} / \mathrm{dl}$ ). Plasma fibrinogen was measured photometrically in accordance to the manufacturer's instructions with citrate plasma (Multifibren ${ }^{\circledR} \mathrm{U}$; Siemens, Germany) (normal 180-350 mg/dl). Plasma WBC was determined using flow cytometry with EDTA plasma (ADVIA Centaur CP; Siemens, Germany) (normal value 4,000-10,000/ $\mu$ l).

Determination of the synovial fluid $A D-1$ levels. Synovial fluid $\alpha-1$ defensin was analyzed using a standard quantitative enzyme immunoassay kit (Human $\alpha$-Defensin 1 Antibody, R\&D Systems BioTechne, Minneapolis, USA/cut-off level $4800 \mathrm{ng} / \mathrm{ml}$ ). The results were given as a standardized signal relative to a tolerance limit value (interpretation values: $<0.9$ aseptic, 0.9-0.99 unspecific, $\geq 1.0$ septic).

After 1 year, we evaluated whether patients showed presence or absence of persistent PJI after the second stage revision using the Delphi criteria (10) and compared these data to the AD-1 test results.

Statistical analysis. To evaluate the accuracy of $\mathrm{AD}-1$ as a diagnostic marker for infection control at 1 year postoperatively, we measured statistical values in comparison to the Delphi criteria as a reference standard. The data were processed with the statistical software package SPSS and Graph Pad Prism 8. Normally distributed continuous data were shown as mean \pm standard deviation (SD) and compared using student's $t$-test. Non-normally distributed continuous data were shown as mean and compared using the Mann-Whitney $U$-test. A $p$-value $<0.05$ was considered statistically significant. To figure out value changes between the first and second stage, the paired samples $t$-test for normally distributed continuous data und the Wilcoxon signed rank test for non-normally distributed continuous data were applied. Sensitivity, specificity, and their $95 \%$ confidence interval (CI) for any cut-off level were calculated. Receiver operating characteristic (ROC) curves were subsequently constructed by mapping true-positive rate (sensitivity) against falsepositive rate ( 1 - specificity) for each test-joint combination. The 
A

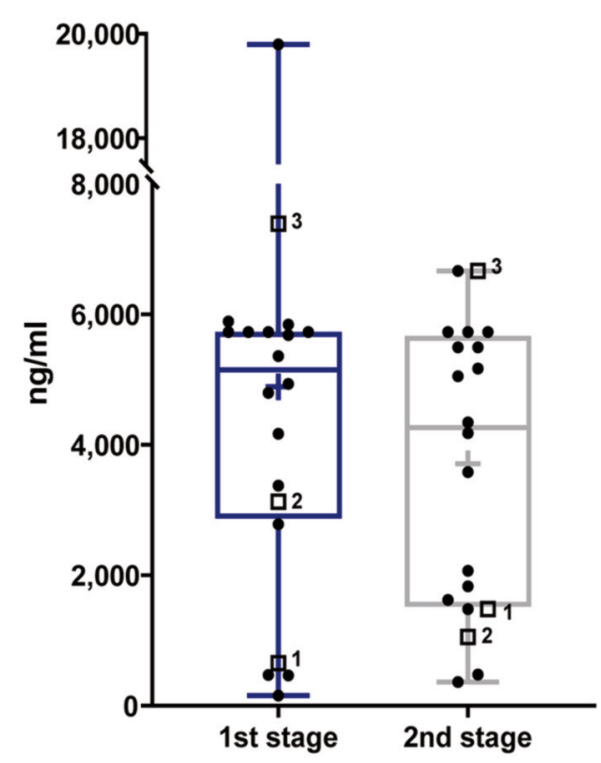

C

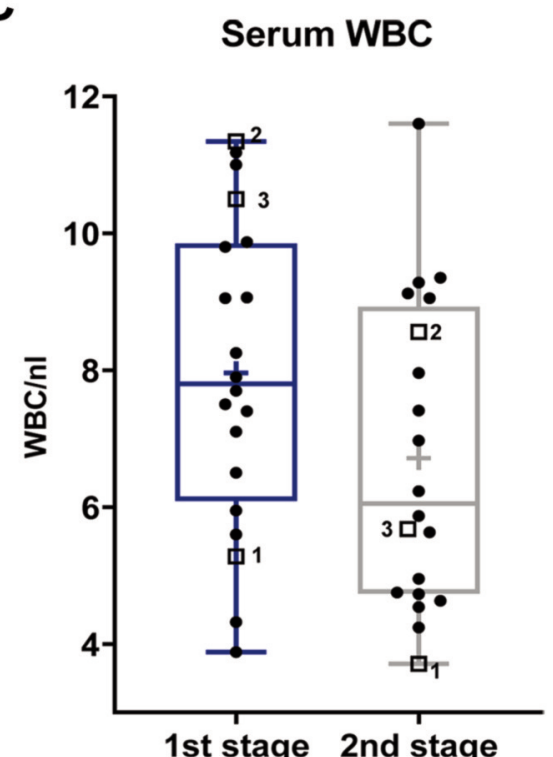

B

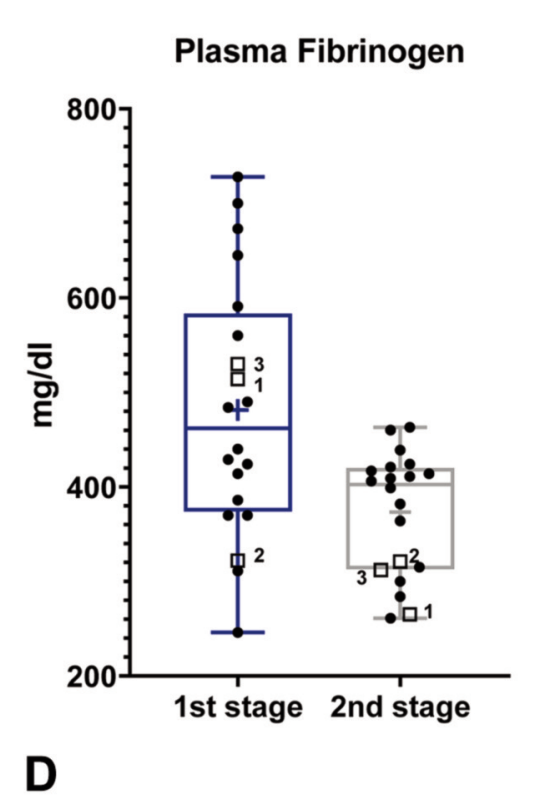

Serum CRP

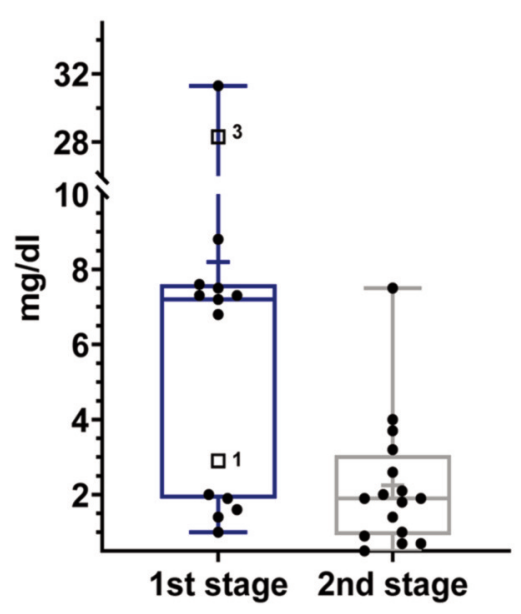

Figure 1. Log-scale dot plots demonstrate the distribution of synovial AD-1 (A), fibrinogen (B), serum WBC (C), and CRP (D), before the first and second stage. The three patients who met the Delphi Criteria at 1-year follow-up are each separately marked with a square and a number, whereas the ones who were not diagnosed as reinfected are tagged with a dot. Regarding serum CRP, only values above the analytical limit of detection $(<0.5 \mathrm{mg} / \mathrm{dl})$ are shown (no patients with persistent PJI).

ROC curve is a graphical statistical tool that illustrates the discriminative effectiveness for a diagnostic test.

\section{Results}

Synovial fluid AD-1 levels and LE test. Before first-stage procedure, the mean AD-1 level was $4,890.99 \mathrm{ng} / \mathrm{ml}$ (range $=154.43-19,796.20 \mathrm{ng} / \mathrm{ml}$ ) and the AD-1 test was positive for PJI $(>4,800 \mathrm{ng} / \mathrm{ml})$ in $55 \%$ of patients $(95 \% \mathrm{CI}=34 \cdot 2-74.2 \%)$. No statistically significant downtrend of synovial AD-1 between the two procedures could be observed ( $p=0.35$; Table I, Figure 1A). At 1-year follow-up, 15 of 20 patients were available, 3 of whom suffered from persistent PJI according to the Delphi Criteria. The AD-1-test only predicted one of three reinfections, whereas in 8 patients who did not meet the criteria, results were false-positive. The overall mean 


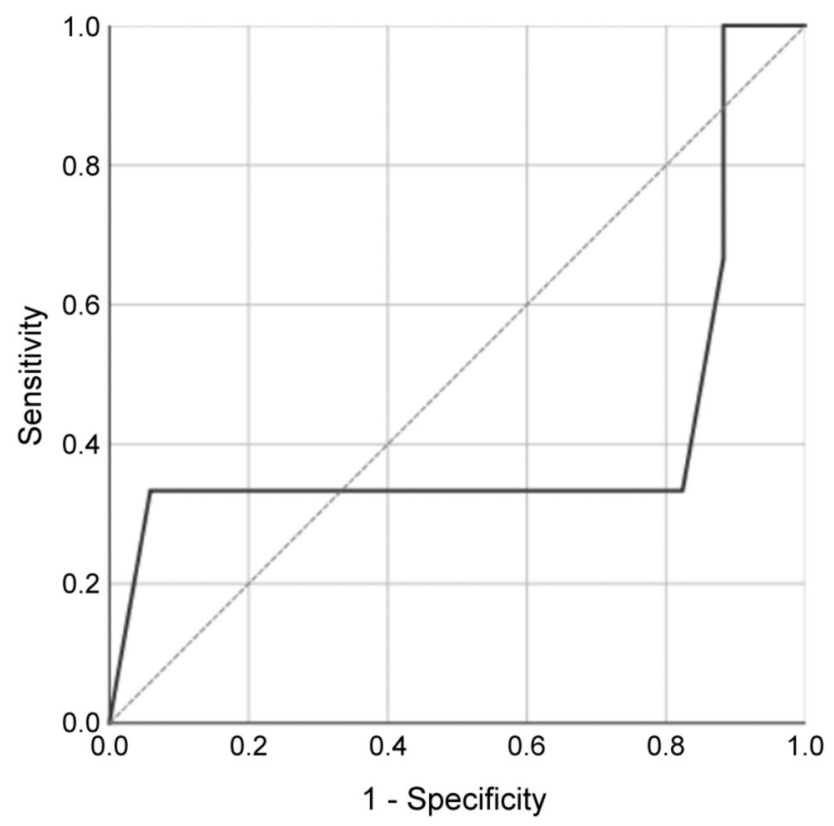

Figure 2. Receiver operating characteristic curve for synovial fluid AD-1.

AD-1 concentration before reimplantation was $3,711.03$ $\mathrm{ng} / \mathrm{ml} \pm 2,174.38 \mathrm{ng} / \mathrm{ml}$. In patients who suffered from reinfection at 1-year follow-up, mean synovial $\mathrm{AD}-1$ level was $3,067.86 \mathrm{ng} / \mathrm{ml} \pm 105.26 \mathrm{ng} / \mathrm{ml}$, whereas in patients who remained infection-free, it was $3,824.53 \mathrm{ng} / \mathrm{ml} \pm 2,074.65 \mathrm{ng} / \mathrm{ml}$. No statistically significant difference between the two groups could be demonstrated ( $p=0.689$ ). Applying $4,800 \mathrm{ng} / \mathrm{ml}$ as a threshold level, sensitivity, specificity and AUC yielded 33.3\% $(95 \% \mathrm{CI}=1.7-88.2 \%), 52.9 \%(95 \% \mathrm{CI}=31.0-73.8 \%)$ and 0.412 (95\% CI=0-0.875), respectively (Table II, Figures $1 \mathrm{~A}$ and 2).

According to the MSIS criteria, we considered (++/+++) a positive LE test result. Prior to first-stage revision (Figure 3), 7 of 19 patients with primary PJI showed a positive LE test (sensitivity $36.84 \% ; 95 \% \mathrm{CI}=19.15-58.96$ ). Of the 3 patients suffering from reinfection, only one had a positive test result at the time of explantation. In one case before the first stage and in two cases before the second stage, the test strip was unreadable due to colour disturbance caused by blood contamination in spite of prior centrifugation. Before second-stage revision, the LE test was negative (negative/trace/one-positive + ) in the remaining 18 patients, including all patients suffering from reinfection (Figure 3 ). A (+) result occurred in only one patient who had not failed at 1-year follow-up. Consequently, the LE test yielded sensitivity and specificity of $0 \%(95 \% \mathrm{CI}=0-56.2 \%)$ and $100 \% \quad(95 \% \mathrm{CI}=79.6-100 \%)$. A statistically significant decrease of semiquantitatively measured LE levels between first and second stage revision could be shown $(p=0.01)$.

\section{Leukocyte Esterase}

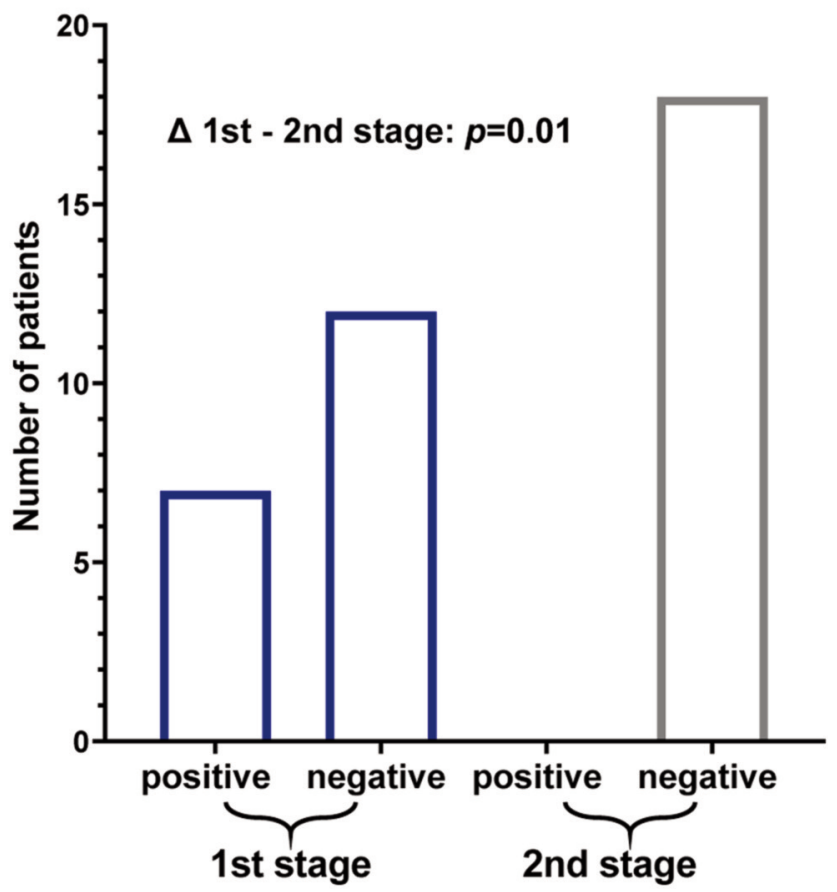

Figure 3. Results of synovial LE testing before the first and second stage.

Serum CRP, WBC and fibrinogen. With regard to blood analysis at the first stage, plasma fibrinogen levels were elevated $(>350 \mathrm{mg} / \mathrm{dl})$ in $85 \%(95 \% \mathrm{CI}=64-94.8 \%)$, whereas only $20 \%(95 \% \mathrm{CI}=8.1-41.6 \%)$ of all patients had increased WBC $(>10,000 / \mu 1)$, including two patients suffering from reinfection after one year in each case. Mean WBC and fibrinogen were $7,959 / \mu \mathrm{l} \pm 2,261 / \mu \mathrm{l}$ and $481.35 \mathrm{md} / \mathrm{dl} \pm 136.69$ $\mathrm{mg} / \mathrm{dl}$ (Table I, Figures $1 \mathrm{~B}$ and C). Preceding reimplantation, mean WBC and fibrinogen were $5,983 / \mu \mathrm{l} \pm 2,439 / \mu \mathrm{l}$ and $299.33 \mathrm{mg} / \mathrm{dl} \pm 30.07 \mathrm{mg} / \mathrm{dl}$ in patients who met the DelphiCriteria at 1-year follow-up, whereas in patients without reinfection, mean WBC and fibrinogen were $6,842 / \mu \mathrm{l} \pm 2,218 / \mu \mathrm{l}$ and $386.41 \mathrm{mg} / \mathrm{dl} \pm 60.88 \mathrm{mg} / \mathrm{dl}$ (Table II, Figures $1 \mathrm{~B}$ and $\mathrm{C})$, respectively. For WBC $(p=0.812)$ and fibrinogen $(p=0.23)$ no statistically significant difference was shown between patients with persistent PJI and patients who did not meet the Delphi Criteria at 1-year follow-up. WBC was normal $(<10,000 / \mu l)$ before reimplantation in 19 patients. Nevertheless, significant downtrends for fibrinogen $(p=0.025)$ and WBC ( $p=0.035)$ between the two stages were determined.

Regarding serum CRP prior to the first stage, 70\% (95\%CI $=51.9-85.5 \%)$ revealed levels above $1 \mathrm{mg} / \mathrm{dl}$ as a MSIS minor criterium cut-off for primary PJI, and among them two patients with reinfection at 1 -year follow-up 
(Figure 1D). Mean serum CRP was $6.15 \mathrm{mg} / \mathrm{dl}$ (range $<0.5$ $31.3 \mathrm{mg} / \mathrm{dl})$. A statistically significant decrease $(p=0.01)$ between the two procedures was observed (Table I, Figure 1D). Before reimplantation, levels were statistically significantly lower $(p=0.04)$, even below the analytical limit of detection $(<0.5 \mathrm{mg} / \mathrm{dl})$ in all patients with reinfection (Table II, Figure 1D). Sixteen of 17 patients who did not meet the Delphi-Criteria at 1-year follow-up had elevated serum-CRP levels (mean $2.11 \mathrm{mg} / \mathrm{dl}, 0.5-7.5 \mathrm{mg} / \mathrm{dl}$ ).

Outcome of PJI treatment. The majority of initial PJIs were caused by staphylococci (50\%). Organisms cultured at first stage procedure are listed in Table III. Synovial fluid and tissue cultures preserved during reimplantation were negative in all patients. Referring to the patients with reinfection at 1year follow-up, one sustained an acute PJI of the hip caused by a different organism (Streptococcus agalactiae) compared to index PJI (Staphylococcus haemolyticus). Since the infection could not be eradicated by further interventions, a persistent fistula was established. Two patients who had already suffered from recurrent PJI before being included in our study were diagnosed with reinfection of the knee 4 and 7 months after reimplantation. They subsequently underwent further two-stage revision arthroplasty and had an antibioticloaded spacer implanted at 1-year follow-up. The causative organisms were unknown. Two of the remaining 12 patients required revision surgery for aseptic reasons within one year. Overall, 8 reported a significant improvement of joint function. Four patients complained about persisting pain, joint stiffness and slight signs of local inflammation. However, further diagnostics had not been initiated at the time of follow-up, so they did not actually meet the Delphi Criteria.

Of the 5 patients not available, 3 patients died within one year for reasons other than infection (an 89-year old woman died at the first postoperative day due to heart failure and two 67-year old men died due to severe internal diseases 2 and 11 months after reimplantation).

\section{Discussion}

It is paramount to detect resolution of infection in a joint previously treated with component explantation and the placement of an antibiotic-loaded spacer. Two-stage revision arthroplasty, accomplished eventually by additional spacer exchanges, is considered the gold standard in the treatment of PJI (8), especially regarding patients suffering from chronic and partially recurrent, polymicrobial or culturenegative PJI, as they were included in our study. Due to the lack of a gold standard to determine successful eradication of PJI in two stage revision arthroplasty with intermittent implantation of a cement spacer, further research is needed.

The rate of culture negative cases in PJI is estimated at $20 \%$ (13). Hoell et al. (2016) examined the value of cultures of synovial fluid before second stage reimplantation. The sensitivity of the synovial cultures was $5 \%(95 \% \mathrm{CI}=0.13$ $24.87)$, with a specificity of $99 \%(95 \% \mathrm{CI}=94.27-99.97)(14)$. Similarly, Boelch et al. identified synovial fluid culture (SFC) as incapable of ruling out persistent infection (sensitivity and specificity: SFC: 4.6 and 94.3\%) (15). Preiniger et al. compared SFC drawn from patients with intermittent spacer due to PJI of the knee before reimplantation to microbiological and histological examination of tissue samples gained intraoperatively, and found that SFC could only predict $21 \%$ of persisting PJIs diagnosed during reimplantation (16). Our results equally suggest the possibility of false-negative results of SFC during reimplantation, although tissue cultures were negative in all our patients as well. One possible reason for this could be bacterial biofilm formation and persistence owing to insufficient sustained release of antibiotics from the spacer and high tolerance to antibiotic therapy (17).

For this reason, surgeons often use a combination of inflammatory markers in serum to rule out persistent infection (18). Regarding CRP, Hoell et al. reported a sensitivity of $42.1 \%$ and a specificity of $84.2 \%$ (cut-off $2.3 \mathrm{mg} / \mathrm{dl}$ ) (14). Similarly, in our study specificity yielded $88.2 \%$ (cut-off 3.85 $\mathrm{mg} / \mathrm{dl}$ ) whereas sensitivity was even lower, since none of the patients who met the Delphi Criteria at 1-year follow-up had CRP-levels above the analytical limit of detection, in contrast to patients who seemed to be infection-free. CRP levels usually normalize physiologically within 2-4 weeks after surgery, ESR is supposed to be elevated more than 3 months postoperatively (19). Stambough et al. found that there were no significant changes in CRP values when comparing time at resection with time at reimplantation (20). Conversely, Kusuma et al. could show that values of CRP decline precipitously after stage one resection arthroplasty. Yet, comparisons between the infection controlled and persistently infected groups before reimplantation revealed no differences (21). Similarly, we observed a significant downtrend of serum CRP in our study, although this also concerned patients defined as reinfected at 1-year follow-up.

Serum WBC is considered a standardized parameter for systemic inflammation. However, its role in diagnosing primary PJI remains elusive. Zmistowski et al. discovered a low correlation between serum and synovial WBC, and considered serum WBC not to be a valuable parameter to detect PJI (22). Contrarily, Yuan et al. showed significantly elevated serum WBC for patients suffering from PJI, yet AUC was moderate (AUC 0.633) (23). Using a threshold of $10,300 \mathrm{WBC} / \mu \mathrm{l}$, Randau et al. observed high specificity (94.5\%; $95 \% \mathrm{CI}=86.4-98.5 \%)$, although sensitivity was as low as $21.3 \%$ (24). According to these findings, sensitivity for elevated WBC $(>10,000 / \mu \mathrm{l})$ in diagnosing primary PJI yielded only $20 \%$ in our study. Considering the diagnostic value of $\mathrm{WBC}$ to detect persistent PJI before reimplantation, 
sufficient data are lacking. In this context, Mühlhofer et al. showed low sensitivity $(9 \%)$ for serum WBC, nevertheless specificity was high (81\%; AUC 0.426) (25). Similarly, $70.6 \%$ specificity was yielded in our study (treshold $8,260 / \mu \mathrm{l}$ ), whereas sensitivity was low. We found a high variety of WBC with normal levels in most patients (AUC 0.373 ). Although we noted a significant downtrend of WBC between the two stages, we also noted this in two of three patients suffering from reinfection.

Fibrinogen plays a key role in the coagulation cascade and in activating inflammatory processes, e.g. as a response to bacterial infection, and turned out to be a valuable biomarker for detecting primary PJI $(26,27)$. In accordance, elevated fibrinogen levels were detected in $85 \%$ of our patients before first-stage revision. Klim et al. and $\mathrm{Wu}$ et al. reported significantly higher levels of fibrinogen in patients suffering from persisting PJI after an antibiotic-loaded spacer had been inserted $(26,28)$. Xu et al. suggested fibrinogen to be a reliable marker for predicting persistent PJI before reimplantation, as AUC was $0.773(95 \% \mathrm{CI}=0.569-0.905)$ and sensitivity and specificity yielded $87.5 \%$ and $62.8 \%$ (cut-off $361 \mathrm{mg} / \mathrm{dl}$ ), respectively, in their study (27). Contrary to this, we observed no differences in serum fibrinogen levels between patients with PJI at 1-year follow-up and the ones we defined as infection-free (AUC 0.157). We detected a significant decrease in fibrinogen levels between the two stages, yet it also occurred in patients suffering from reinfection at 1-year follow-up.

Based on our results as well as on findings in literature, inflammatory blood parameters are likely influenced by a variety of systemic comorbidities and are not suitable to detect reinfection (26).

Therefore, the search for reliable values to rule out persistent infection was enhanced by testing synovial biomarkers and culture methods. Frangiamore et al. could not find any synovial biomarker to be reliable to rule out infection at the time of reimplantation. However, they found a downtrend between the two stages that may provide an important guide for clinicians to monitor treatment response (29). Leukocyte esterase (LE) is an enzyme secreted by activated polymorphonuclear neutrophils (PMN), which are key players in bacterial infection, and is considered a promising biomarker for primary PJI. LE can be detected semi-quantitively by applying almost any body fluid on a colorimetric strip test. Guenther et al. reported sensitivity and specificity of $100 \%$ and $96.5 \%$, respectively, for LE as a diagnostic test for primary PJI (30). Similarly, Kheir et al. found LE strip test to be a good predictor of persistent infection in patients undergoing reimplantation. In 77 patients, the LE strip test was performed before reimplantation and the mean follow-up was 1.76 years. While specificity and positive predictive value showed values of $100 \%$, sensitivity, negative predictive value and AUC were only $26.3 \%, 87.5 \%$ and 0.632 , respectively (31). In our study, sensitivity of the LE test was low $(36.84 \%)$ for detecting primary PJI. Nevertheless, we observed a notable downtrend of test results between the two stages. Before reimplantation, we could not show any positive (++) results, neither in patients with persistent PJI nor in patients who did not meet the Delphi Criteria at 1-year follow-up. We rather recommend using the LE test strip as a rule-out test for primary PJI than for persistent infection before second-stage revision.

In diagnosing primary $\mathrm{PJI}$, the $\mathrm{AD}-1$ immunoassay is considered to be a reliable tool (1). AD-1 is an antimicrobial peptide secreted by activated neutrophils in response to the presence of pathogens, e.g. bacteria (32). Deirmengian et al. revealed superiority of $\mathrm{AD}-1$ over LE test concerning sensitivity (100\% vs. 69\%) and specificity of $100 \%$ for both tests (1). Bonanzinga et al. reported that accuracy of the AD1 test was not impaired by concomittant metallosis and autoimmune diseases in patients with primary PJI (32). Deirmegian et al. confirmed these results and furthermore, observed that diagnostic accuracy of $\mathrm{AD}-1$ is rarely influenced by virulence and gram staining of pathogens (33). Shahi et al. investigated if the AD-1 test results were affected by prior administration of antibiotics within 2 weeks before joint aspiration and could not find any decrease in sensitivity $(100 \%$; $95 \% \mathrm{CI}=88.4-100 \%)$, in contrast to CRP and SFC (34). For these reasons, we decided that synovial AD-1 could be a promising biomarker to rule out persistent infection even if an antibiotic-loaded spacer had been implanted.

To the best of our knowledge, only one study by Samuel et al. evaluated the diagnostic validity of synovial AD-1 in order to detect persistent PJI after spacer insertion. In 69 patients, synovial AD-1 levels were determined at time of reimplantation and compared to the outcome determined by the Delphi Criteria at minimum 1-year follow-up. Samuel et al. reported a fairly low sensitivity $(7 \% ; 95 \% \mathrm{CI}=0.2-34 \%)$, since 13 of 14 patients (20.2\%) who were identified as suffering from PJI at 1-year follow-up had false-negative results. However, specificity was higher than that in our study $(89 \%, 95 \% \mathrm{CI}=78-96 \%$; AUC $0.5,95 \% \mathrm{CI}=0.3-0,6)$, as only 6 patients who did not meet the Delphi Criteria at 1year follow-up showed false-positive results (9). In our study, sensitivity of synovial AD-1 for the diagnosis of primary PJI was limited, since in only $55 \%$ of patients AD1 test results were positive. One possible reason for this finding may be a high rate of low-grade-PJI, suggesting that neutrophil AD-1 secretion could indeed depend on the virulence of the causing organisms. We then noted no statistically significant decrease in synovial AD-1 levels, but increased false-positive results $(47.1 \%)$ before reimplantation. On the one hand, this could be explained by a higher number of patients who suffered from PJI at 1-year follow-up, but had not undergone further diagnostics at this time and thus, were defined as infection-free. On the other 
hand, the release of $\mathrm{AD}-1$ into the synovial fluid could be increased regardless of infection. Singh et al. observed that PMMA particles released from PMMA spacers due to abrasion may have local immunomodulating effects, as they induced immunocytological changes in periprosthetic tissue (35). Human alpha-defensins, among them AD-1, not only play a crucial role in antimicrobial defense, but also in a variety of physiological processes, e.g. cell migration and wound healing (36). Further research is needed to understand the local immunological reactions to PMMAparticles released from spacers and to consequently discover biomarkers which are not affected by these processes.

An unknown rate of recurrent instead of persistent infections may explain the unexpectedly low sensitivity of AD-1 and also of the LE test in predicting PJI at 1-year follow-up, since it is likely that patients who show low AD1 levels at reimplantation are infection-free at this time and sustain recurrent PJI during the surgery or at any time postoperatively. In the current literature, it seems to be a common phenomenon that a non-negligible proportion of reinfections are not caused by the index organism and thus, cannot directly be defined as persistent PJI. Zmistowski et al. revealed that in only $31.5 \%$ of patients suffering from reinfection the same organisms were isolated at treatment failure (37). Samuel et al. showed a reinfection-rate of $20.3 \%$ at 1-year follow-up, and identical organisms compared to index PJI in only $14.3 \%$ (9). In our study, reinfection rate was $15 \%$ and eventually higher. Two of three patients who met the Delphi Criteria at 1-year followup had a negative AD-1 test result. In one of them, PJI seemed to be successfully eradicated. However, the patient developed symptoms of acute PJI after reimplantation. An organism different to the one isolated at index PJI could be detected. The other patient perhaps suffered from persistent infection (organism of reinfection unknown), since he developed subtle symptoms several months after reimplantation and originally had a recurrent low-grade PJI he was already revised for in the past. Nevertheless, his negative test result perhaps occurred due to either a very low number of vital pathogens locally present during reimplantation or another recurrent infection after an initially infection-free period. These findings underline that patients undergoing treatment of PJI are prone to acquire recurrent infection, especially patients who suffer from an increased number of comorbidities (37).

Our study has several limitations. Unfortunately, we could only identify a low number of patients who met our inclusion criteria. Clinical trials including statistical relevant patient numbers are required to confirm our results. Moreover, we could not record the species of organisms responsible for reinfection in two patients. It is reasonable to suggest that a higher number of patients reporting persistent joint pain and stiffness suffered from PJI at 1-year follow-up, however, they did not meet the Delphi Criteria since further diagnostics had not been initiated. To detect the real midterm failure rate and consequences of two-stage exchange, we plan to carry out another 5-year follow-up. In contrast to a large number of studies, we avoided an "antibiotic holiday" period of at least 2 weeks before gaining synovial fluid for microbiological investigation as well as LE and AD-1 testing. We do not believe that this has significantly impaired our results, since AD-1 has been shown not to be influenced by prior antibiotic administration (34) and low sensitivities for SFC were reported in studies where antibiotics were withheld before joint aspiration as well. Regarding the Proceedings of International Consensus on Orthopedic Infections, it was not clearly recommended to carry out antibiotic holiday due to the possibility of development of resistance to antibiotics before reimplantation (38).

In contrast to a large amount of studies carried out on this topic, we decided to use the Delphi Criteria after 1 year to determine if patients suffer from reinfection instead of a variation of the MSIS criteria on the basis of diagnostic findings achieved during reimplantation, as the patient's health state after reimplantation is more relevant to evaluate treatment success. Frangiamore et al. and Samuel et al. reported low sensitivity of the MSIS criteria concerning the diagnosis of reinfection $(9,29)$. One explanation may be that consistent cut-off levels for parameters included into the minor MSIS criteria before reimplantation are missing. Conversely, the multidimensional Delphi Criteria also include any subsequent surgical intervention due to infection (10), i.e. recurrent infections by organisms other than those isolated at index PJI as treatment failure, which may be an underestimated complication of PJI.

In conclusion, biomarkers in synovial fluid may have limited value to surely determine the outcome of PJI. They could, however, be useful to predict persistent infection before reimplantation. Since $\mathrm{AD}-1$ and $\mathrm{LE}$ seem to be unsuitable for this purpose, further biomarkers should be investigated to finally develop a test reliably confirming total eradication of PJI.

\section{Conflicts of Interest}

There are no financial or other interests regarding the submitted manuscript that might be construed as a conflict of interest.

\section{Authors' Contributions}

MJ supervised the clinical trial as head of the orthopedic department. $\mathrm{AB}$ designed the study. $\mathrm{CB}$ recruited and interviewed patients at 1-year follow-up. MJ and AW performed joint aspiration. Sample analysis was carried out by HE. DW and AW were responsible for data analysis and generation of graphics. $\mathrm{AB}$ and $\mathrm{CB}$ wrote and revised the manuscript. All Authors ensured that they are in agreement with form and contents of the manuscript. 


\section{Acknowledgements}

Special thanks go to Alexandra Kornowski who performed the immunoassays.

\section{References}

1 Deirmengian C, Kardos K, Kilmartin P, Cameron A, Schiller K, Booth RE, Jr. and Parvizi J: The alpha-defensin test for periprosthetic joint infection outperforms the leukocyte esterase test strip. Clin Orthop Relat Res 473(1): 198-203, 2015. PMID: 24942960. DOI: 10.1007/s11999-014-3722-7

2 Gundtoft PH, Pedersen AB, Varnum C and Overgaard S: Increased mortality after prosthetic joint infection in primary tha. Clin Orthopaed Relat Res 475(11): 2623-2631, 2017. PMID: 28236084. DOI: 10.1007/s11999-017-5289-6

3 Parvizi J, Tan TL, Goswami K, Higuera C, Della Valle C, Chen $\mathrm{AF}$ and Shohat N: The 2018 definition of periprosthetic hip and knee infection: An evidence-based and validated criteria. J Arthroplasty 33(5): 1309-1314.e1302, 2018. PMID: 29551303. DOI: $10.1016 /$ j.arth.2018.02.078

4 Bingham J, Clarke H, Spangehl M, Schwartz A, Beauchamp C and Goldberg B: The alpha defensin-1 biomarker assay can be used to evaluate the potentially infected total joint arthroplasty. Clin Orthop Relat Res 472(12): 4006-4009, 2014. PMID: 25256621. DOI: 10.1007/s11999-014-3900-7

5 Deirmengian C, Kardos K, Kilmartin P, Cameron A, Schiller K and Parvizi J: Diagnosing periprosthetic joint infection: Has the era of the biomarker arrived? Clin Orthop Relat Res 472(11): 3254-3262, 2014. PMID: 24590839. DOI: 10.1007/s11999-0143543-8

6 Deirmengian C, Kardos K, Kilmartin P, Cameron A, Schiller K and Parvizi J: Combined measurement of synovial fluid $\alpha-$ defensin and c-reactive protein levels: Highly accurate for diagnosing periprosthetic joint infection. J Bone Joint Surg Am 96(17): 1439-1445, 2014. PMID: 25187582. DOI: 10.2106/ jbjs.m.01316

7 Kunutsor SK, Whitehouse MR, Blom AW, Board T, Kay P, Wroblewski BM, Zeller V, Chen SY, Hsieh PH, Masri BA, Herman A, Jenny JY, Schwarzkopf R, Whittaker JP, Burston B, Huang R, Restrepo C, Parvizi J, Rudelli S, Honda E, Uip DE, Bori G, Muñoz-Mahamud E, Darley E, Ribera A, Cañas E, Cabo J, Cordero-Ampuero J, Redó MLS, Strange S, Lenguerrand E, Gooberman-Hill R, Webb J, MacGowan A, Dieppe P, Wilson M and Beswick AD: One- and two-stage surgical revision of periprosthetic joint infection of the hip: A pooled individual participant data analysis of 44 cohort studies. Eur J Epidemiol 33(10): 933-946, 2018. PMID: 29623671. DOI: 10.1007/s10654018-0377-9

8 Cooper HJ and Della Valle CJ: The two-stage standard in revision total hip replacement. Bone Joint J 95-b(11 Suppl A): 84-87, 2013. PMID: 24187360. DOI: 10.1302/0301620x.95b11.32906

9 Samuel LT, Sultan AA, Kheir M, Villa J, Patel P, Parvizi J and Higuera CA: Positive alpha-defensin at reimplantation of a twostage revision arthroplasty is not associated with infection at 1 year. Clin Orthop Relat Res 477(7): 1615-1621, 2019. PMID: 30811358. DOI: 10.1097/corr.0000000000000620

10 Diaz-Ledezma C, Higuera CA and Parvizi J: Success after treatment of periprosthetic joint infection: A delphi-based international multidisciplinary consensus. Clin Orthop Relat Res 471(7): 2374-2382, 2013. PMID: 23440616. DOI: 10.1007/ s11999-013-2866-1

11 Kheir MM, Tan TL, George J, Higuera CA, Maltenfort MG and Parvizi J: Development and evaluation of a prognostic calculator for the surgical treatment of periprosthetic joint infection. $J$ Arthroplasty 33(9): 2986-2992.e2981, 2018. PMID: 29885971. DOI: $10.1016 /$ j.arth.2018.04.034

12 Kurd MF, Ghanem E, Steinbrecher J and Parvizi J: Two-stage exchange knee arthroplasty: Does resistance of the infecting organism influence the outcome? Clin Orthop Relat Res 468(8): 2060-2066, 2010. PMID: 20300903. DOI: 10.1007/s11999-0101296-6

13 Bjerke-Kroll BT, Christ AB, McLawhorn AS, Sculco PK, JulesElysée KM and Sculco TP: Periprosthetic joint infections treated with two-stage revision over 14 years: An evolving microbiology profile. J Arthroplasty 29(5): 877-882, 2014. PMID: 24199997. DOI: $10.1016 /$ j.arth.2013.09.053

14 Hoell S, Moeller A, Gosheger G, Hardes J, Dieckmann R and Schulz D: Two-stage revision arthroplasty for periprosthetic joint infections: What is the value of cultures and white cell count in synovial fluid and crp in serum before second stage reimplantation? Arch Orthop Trauma Surg 136(4): 447-452, 2016. PMID: 26757939. DOI: 10.1007/s00402-015-2404-6

15 Boelch SP, Weissenberger M, Spohn F, Rudert M and Luedemann M: Insufficient sensitivity of joint aspiration during the two-stage exchange of the hip with spacers. J Orthop Surg Res 13(1): 7, 2018. PMID: 29321073. DOI: 10.1186/s13018-017-0703-Z

16 Preininger B, Janz V, von Roth P, Trampuz A, Perka CF and Pfitzner T: Inadequacy of joint aspiration for detection of persistent periprosthetic infection during two-stage septic revision knee surgery. Orthopedics 40(4): 231-234, 2017. PMID: 28418574. DOI: 10.3928/01477447-20170411-04

17 Ma D, Shanks RMQ, Davis CM, 3rd, Craft DW, Wood TK, Hamlin BR and Urish KL: Viable bacteria persist on antibiotic spacers following two-stage revision for periprosthetic joint infection. J Orthop Res 36(1): 452-458, 2018. PMID: 28543707. DOI: $10.1002 /$ jor.23611

18 Mont MA, Waldman BJ and Hungerford DS: Evaluation of preoperative cultures before second-stage reimplantation of a total knee prosthesis complicated by infection. A comparisongroup study. J Bone Joint Surg Am 82(11): 1552-1557, 2000. PMID: 11097443. DOI: 10.2106/00004623-200011000-00006

19 Ghanem E, Azzam K, Seeley M, Joshi A and Parvizi J: Staged revision for knee arthroplasty infection: What is the role of serologic tests before reimplantation? Clin Orthop Relat Res 467(7): 1699-1705, 2009. PMID: 19241115. DOI: $10.1007 /$ s11999-009-0742-9

20 Stambough JB, Curtin BM, Odum SM, Cross MB, Martin JR and Fehring TK: Does change in esr and crp guide the timing of two-stage arthroplasty reimplantation? Clin Orthop Relat Res 477(2): 364-371, 2019. PMID: 30566107. DOI: 10.1097/01. blo.0000533618.31937.45

21 Kusuma SK, Ward J, Jacofsky M, Sporer SM and Della Valle $\mathrm{CJ}$ : What is the role of serological testing between stages of twostage reconstruction of the infected prosthetic knee? Clin Orthop Relat Res 469(4): 1002-1008, 2011. PMID: 20941647. DOI: 10.1007/s11999-010-1619-7

22 Zmistowski B, Restrepo C, Huang R, Hozack WJ and Parvizi J: Periprosthetic joint infection diagnosis: A complete 
understanding of white blood cell count and differential. J Arthroplasty 27(9): 1589-1593, 2012. PMID: 22543180. DOI: 10.1016/j.arth.2012.03.059

23 Yuan K, Li WD, Qiang Y and Cui ZM: Comparison of procalcitonin and c-reactive protein for the diagnosis of periprosthetic joint infection before revision total hip arthroplasty. Surg Infect (Larchmt) 16(2): 146-150, 2015. PMID: 25658716. DOI: $10.1089 /$ sur.2014.034

24 Randau TM, Friedrich MJ, Wimmer MD, Reichert B, Kuberra D, Stoffel-Wagner B, Limmer A, Wirtz DC and Gravius S: Interleukin-6 in serum and in synovial fluid enhances the differentiation between periprosthetic joint infection and aseptic loosening. PLoS One 9(2): e89045, 2014. PMID: 24586496. DOI: 10.1371 /journal.pone.0089045

25 Mühlhofer HML, Knebel C, Pohlig F, Feihl S, Harrasser N, Schauwecker J and von Eisenhart-Rothe R: Synovial aspiration and serological testing in two-stage revision arthroplasty for prosthetic joint infection: Evaluation before reconstruction with a mean follow-up of twenty seven months. Int Orthop 42(2): 265-271, 2018. PMID: 29243060. DOI: 10.1007/s00264-0173700-2

26 Klim SM, Amerstorfer F, Gruber G, Bernhardt GA, Radl R, Leitner L, Leithner A and Glehr M: Fibrinogen - a practical and cost efficient biomarker for detecting periprosthetic joint infection. Sci Rep 8(1): 8802, 2018. PMID: 29892047. DOI: 10.1038/s41598-018-27198-3

27 Xu C, Qu PF, Chai W, Li R and Chen JY: Plasma fibrinogen may predict persistent infection before reimplantation in twostage exchange arthroplasty for periprosthetic hip infection. J Orthop Surg Res 14(1): 133, 2019. PMID: 31088508. DOI: 10.1186/s13018-019-1179-9

$28 \mathrm{Wu}$ H, Meng Z, Pan L, Liu H, Yang X and Yongping C: Plasma fibrinogen performs better than plasma d-dimer and fibrin degradation product in the diagnosis of periprosthetic joint infection and determination of reimplantation timing. J Arthroplasty 35(8): 2230-2236, 2020. PMID: 32376167. DOI: 10.1016/j.arth.2020.03.055

29 Frangiamore SJ, Siqueira MB, Saleh A, Daly T, Higuera CA and Barsoum WK: Synovial cytokines and the msis criteria are not useful for determining infection resolution after periprosthetic joint infection explantation. Clin Orthop Relat Res 474(7): 16301639, 2016. PMID: 26821163. DOI: 10.1007/s11999-016-4710-х

30 Guenther D, Kokenge T, Jacobs O, Omar M, Krettek C, Gehrke $\mathrm{T}$, Kendoff $\mathrm{D}$ and Haasper $\mathrm{C}$ : Excluding infections in arthroplasty using leucocyte esterase test. Int Orthop 38(11): 2385-2390, 2014. PMID: 25027977. DOI: 10.1007/s00264-0142449-0
31 Kheir MM, Ackerman CT, Tan TL, Benazzo A, Tischler EH and Parvizi J: Leukocyte esterase strip test can predict subsequent failure following reimplantation in patients with periprosthetic joint infection. J Arthroplasty 32(6): 1976-1979, 2017. PMID: 30811358. DOI: 10.1016/j.arth.2017.01.031

32 Bonanzinga T, Zahar A, Dütsch M, Lausmann C, Kendoff D and Gehrke T: How reliable is the alpha-defensin immunoassay test for diagnosing periprosthetic joint infection? A prospective study. Clin Orthop Relat Res 475(2): 408-415, 2017. PMID: 27343056. DOI: 10.1007/s11999-016-4906-0

33 Deirmengian C, Kardos K, Kilmartin P, Gulati S, Citrano P and Booth RE Jr.: The alpha-defensin test for periprosthetic joint infection responds to a wide spectrum of organisms. Clin Orthop Relat Res 473(7): 2229-2235, 2015. PMID: 25631170. DOI: 10.1007/s11999-015-4152-x

34 Shahi A, Parvizi J, Kazarian GS, Higuera C, Frangiamore S, Bingham J, Beauchamp C, Valle CD and Deirmengian C: The alpha-defensin test for periprosthetic joint infections is not affected by prior antibiotic administration. Clin Orthop Relat Res 474(7): 1610-1615, 2016. PMID: 26864855. DOI: 10.1007/ s11999-016-4726-2

35 Singh G, Deutloff N, Maertens N, Meyer H, Awiszus F, Feuerstein B, Roessner A and Lohmann CH: Articulating polymethylmethacrylate (pmma) spacers may have an immunomodulating effect on synovial tissue. Bone Joint J 98b(8): 1062-1068, 2016. PMID: 27482018. DOI: 10.1302/0301620x.98b8.36663

36 Frasca L and Lande R: Role of defensins and cathelicidin 1137 in auto-immune and auto-inflammatory diseases. Curr Pharm Biotechnol 13(10): 1882-1897, 2012. PMID: 22250708. DOI: $10.2174 / 138920112802273155$

37 Zmistowski B, Tetreault MW, Alijanipour P, Chen AF, Della Valle CJ and Parvizi J: Recurrent periprosthetic joint infection: Persistent or new infection? J Arthroplasty 28(9): 1486-1489, 2013. PMID: 23587491. DOI: 10.1016/j.arth.2013.02.021

38 Aalirezaie A, Bauer TW, Fayaz H, Griffin W, Higuera CA, Krenn V, Krenn V, Molano M, Moojen DJ, Restrepo C, Shahi A, Shubnyakov I, Sporer S, Tanavalee A, Teloken M and Velázquez Moreno JD: Hip and knee section, diagnosis, reimplantation: Proceedings of international consensus on orthopedic infections. J Arthroplasty 34(2s): S369-s379, 2019. PMID: 30343965. DOI: 10.1016/j.arth.2018.09.021

Received November 23, 2020

Revised December 6, 2020

Accepted December 7, 2020 